\title{
Maturing thalamocortical functional connectivity across development
}

\author{
Damien A. Fair ${ }^{1 *}$, Deepti Bathula ${ }^{1}$, Kathryn L. Mills ${ }^{1}$,Taciana G. Costa Dias ${ }^{1}$, Michael S. Blythe ${ }^{1}$, Dongyang \\ Zhang $^{2}$, Abraham Z. Snyder ${ }^{2}$, Marcus E. Raichle ${ }^{2}$, Alexander A. Stevens ${ }^{1,3}$, JoelT. Nigg ${ }^{1,3}$ and Bonnie J. Nagel ${ }^{1,3 *}$ \\ 1 Department of Psychiatry, Oregon Health and Science University, Portland, OR, USA \\ 2 Department of Radiology, Washington University School of Medicine, St. Louis, MO, USA \\ ${ }^{3}$ Department of Behavioral Neuroscience, Oregon Health and Science University, Portland, OR, USA
}

\section{Edited by:}

Lucina Q. Uddin, Stanford University, USA

\section{Reviewed by:}

Beatriz Luna,

University of Pittsburgh, USA

Daniel Margulies,

Max Planck Institute, Germany

*Correspondence:

Damien A. Fair, Department of Psychiatry, Oregon Health and Science University, 3181 SW Sam Jackson Park Road UHN80R1, Portland, OR 97239,

USA.

e-mail: damien.fair@aya.yale.edu; Bonnie J. Nagel, Department of

Psychiatry, Oregon Health and Science University, 3181 SW Sam Jackson Park Road DC7P, Portland, Oregon 97239, USA.

e-mail:nagelb@ohsu.edu
Recent years have witnessed a surge of investigations examining functional brain organization using resting-state functional connectivity MRI (rs-fcMRI). To date, this method has been used to examine systems organization in typical and atypical developing populations. While the majority of these investigations have focused on cortical-cortical interactions, cortical-subcortical interactions also mature into adulthood. Innovative work by Zhang et al. (2008) in adults have identified methods that utilize rs-fcMRI and known thalamo-cortical topographic segregation to identify functional boundaries in the thalamus that are remarkably similar to known thalamic nuclear grouping. However, despite thalamic nuclei being well formed early in development, the developmental trajectory of functional thalamo-cortical relations remains unexplored. Thalamic maps generated by rs-fcMRI are based on functional relationships, and should modify with the dynamic thalamo-cortical changes that occur throughout maturation. To examine this possibility, we employed a strategy as previously described by Zhang et al. to a sample of healthy children, adolescents, and adults. We found strengthening functional connectivity of the cortex with dorsal/anterior subdivisions of the thalamus, with greater connectivity observed in adults versus children. Temporal lobe connectivity with ventral/midline/posterior subdivisions of the thalamus weakened with age. Changes in sensory and motor thalamo-cortical interactions were also identified but were limited. These findings are consistent with known anatomical and physiological cortical-subcortical changes over development. The methods and developmental context provided here will be important for understanding how cortical-subcortical interactions relate to models of typically developing behavior and developmental neuropsychiatric disorders.

\section{INTRODUCTION}

Recent years have witnessed a surge of investigations examining brain function and organization using the relatively new technique of resting-state functional connectivity MRI (rs-fcMRI) (Biswal et al., 1995). rs-fcMRI measures correlate, low frequency (usually $<0.1 \mathrm{~Hz}$ ) blood oxygenation level dependent (BOLD) fluctuations between brain regions while subjects are at rest, not performing goaldirected tasks (Biswal et al., 1995; Fox et al., 2005; Fair et al., 2007a,b, 2008; Fox and Raichle, 2007). By cross correlating the BOLD signal time series between different regions or voxels, one can determine which regions are 'functionally connected' (see Friston et al., 1993; Lee et al., 2003 for specific definition). To date, this method has been used in several domains to examine systems-level brain organization in typical and atypical populations (Biswal et al., 1995; Fox et al., 2006; Hampson et al., 2006; Andrews-Hanna et al., 2007; Dosenbach et al., 2007; Fair et al., 2007a, 2008, 2009; Greicius et al., 2007; Seeley et al., 2007; Uddin et al., 2008; Church et al., 2009).

Recent work has shown that rs-fcMRI is also quite useful for studying the maturation of functional brain networks. This work has led to key insights regarding typical and atypical brain development (Fair et al., 2007a, 2008, 2009; Fransson et al., 2007; Kelly et al., 2009; Supekar et al., 2009). Whereas the majority of these inves- tigations have focused on cortical-cortical interactions, subcortical structures and their cortical interactions also develop during childhood and adolescence (Jones, 2007). Indeed, recent rs-fcMRI studies have suggested distinct developmental changes that occur between cortical and subcortical structures (Fair et al., 2007a, 2009; Kelly et al., 2009; Supekar et al., 2009). Thus, important insights regarding brain development are likely to emerge from additional examination of cortical-subcortical functional relationships.

Knowledge concerning the developmental trajectory of rs-fcMRI is especially lacking with regards to the thalamus, a key structure for nearly all brain operations. Innovative work by Zhang et al. (2008, 2009) in adults has recently used rs-fcMRI to map thalamo-cortical interactions. This methodology utilizes known cortical connectional anatomy (Alexander and Crutcher, 1990; Jones, 2007) to identify functional boundaries in the thalamus and other subcortical structures. In this procedure (Zhang et al., 2008), regions of interest (ROIs) are identified that encompass major subdivisions of the cortex (see Figure 1). The average spontaneous signal generated in each cortical ROI is then correlated with all of the voxels in the thalamus. Using a 'winner take all' strategy, where the cortical subdivision that correlates strongest with a given voxel 'wins,' Zhang et al. partitioned the thalamus into distinct subdivisions 


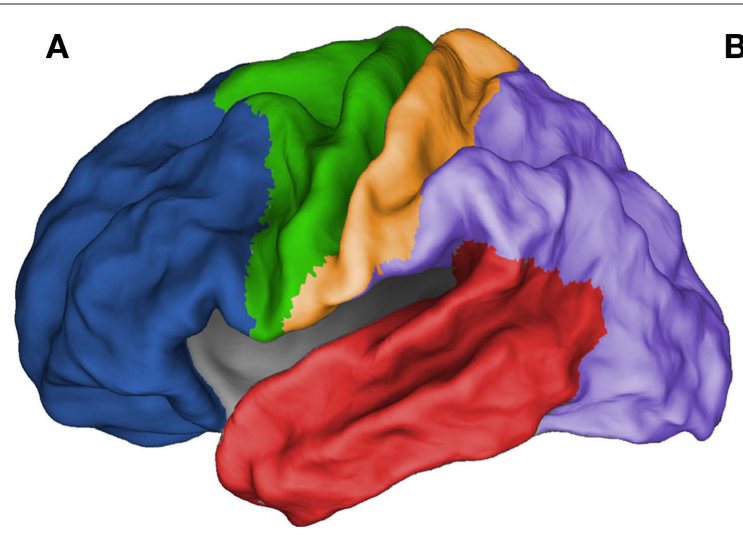

FIGURE 1 |The 'winner take all' strategy for identifying subdivisions of the thalamus (Zhang et al., 2008), utilizes known thalamocortical topographic segregation (Alexander and Crutcher, 1990; Jones, 2007) to identify functional boundaries in the thalamus. (A) Regions of interest (ROls) used for the current analysis. These regions are composed of five disjoint cortical subdivisions, which include the prefrontal cortex (blue), motor/premotor (green), somatosensory (yellow), parietal/occipical cortex (purple), and temporal corex (red). (B) The average spontaneous signal generated from each cortical $\mathrm{ROI}$ is then correlated with all of
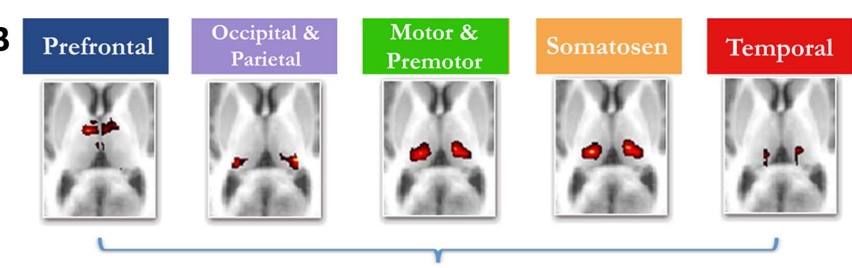

C
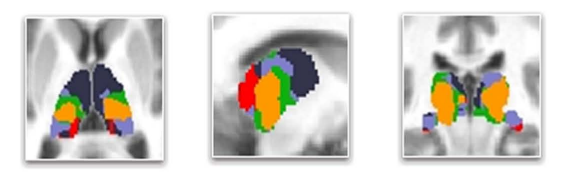

the voxels in the thalamus. This creates five voxelwise statistical maps of the thalamus. (C) The strength of connectivity is then compared for each cortical ROI within each voxel. The cortical subdivision that correlates strongest with any given voxel 'wins.' The given voxel is then assigned the color of the winning cortical ROI. The resulting thalamic subdivisions are in substantial agreement with known thalamic nuclear grouping based on postmortem human studies (Morel, 2007; Mai et al., 2008) and anatomical track-tracing data from other mammalian species (Nieuwenhuys, 1988; Webster et al., 1995; Jones, 2007)
(Figure 1). The spatial organization of these thalamic subdivisions is in substantial agreement with known thalamic nuclear grouping based on postmortem human studies (Morel, 2007; Mai et al., 2008) and anatomical track-tracing data from other mammalian species (Nieuwenhuys, 1988; Webster et al., 1995; Jones, 2007). The rs-fcMRI results also are remarkably similar to tract-tracing results based on diffusion tensor imaging (DTI) (Behrens et al., 2003; Johansen-Berg et al., 2005; Zhang et al., 2009).

While anatomically distinct nuclear groups are well formed within the thalamus early in development (Jones, 2007), it is unknown whether thalamo-cortical fcMRI is the same in children and adults. Because the thalamic maps generated by fcMRI are based on functional relationships, we hypothesized they should not mimic organization found in adulthood, but should track the dynamic thalamocortical changes that are believed to occur throughout maturation (e.g., the pruning of temporal-thalamic contacts, and increased frontal-subcortical coherence over age) (Giedd et al., 1999; Jones, 2007; Galinanes et al., 2009). This developmental characterization between thalamus and cortex has the potential to lay the groundwork for a better understanding of how cortical-subcortical interactions contribute to the shift from reflexive, stimulus-bound behavior in childhood, to the goal-directed and more flexible functioning found in adulthood. It will also provide the neural context necessary to examine how cortical-thalamic interactions relate to prominent models of several developmental neuropsychiatric disorders. Hence, we employed a strategy previously detailed by Zhang et al. to study correlated spontaneous brain activity between the cortex and the thalamus in healthy children, adolescents, and adults.

\section{MATERIALS AND METHODS PARTICIPANTS}

Participants were recruited through a combination of public advertisements, county mailings, and via the Oregon Health \& Sciences University local outreach systems. Participants were screened with a series of interviews and questionnaires for inclusion. Informed consent was obtained from all subjects in accordance with the guidelines and approval of the Oregon Health \& Science University Human Investigation Review Board. A total of 52 subjects (17 aged 7-9; 21 aged 11-16; 14 aged 19-32) were included in the study (see Table 1; Table S1 in Supplementary Material). All participants were free of major sensory, medical, neurological, or psychiatric (including substance abuse) illness and had normal-range intelligence.

\section{DATA ACQUISITION AND PROCESSING}

Participants were scanned using a 3.0 Tesla Siemens Magnetom Tim Trio scanner with a twelve-channel head-coil at the OHSU Advanced Imaging Research Center (AIRC). One high resolution T1weighted MPRAGE sequence $($ orientation $=$ sagittal, $\mathrm{TE}=3.58 \mathrm{~ms}$, $\mathrm{TR}=2300 \mathrm{~ms}, 256 \times 256$ matrix, resolution $=(1 \mathrm{~mm})^{3}, 1$ average, total scan time $=9$ min $14 \mathrm{~s}$ ) was collected. Blood-oxygen level dependent (BOLD)-weighted functional imaging was collected in an oblique plane (parallel to the ACPC line) using $\mathrm{T} 2^{*}$-weighted echo-planar imaging. Resting data from adult participants were originally collected as part of a separate study. As such, acquisition parameters were slightly different for adults and children/ adolescents (adults: $\mathrm{TR}=2000 \mathrm{~ms}, \mathrm{TE}=30 \mathrm{~ms}$, flip angle $=90^{\circ}$, FOV $=240 \mathrm{~mm}$, 36 slices, slice thickness $=3.5 \mathrm{~mm}$ in-plane resolution $=3.75 \mathrm{~mm}^{2}$; children: $\mathrm{TR}=2000 \mathrm{~ms}$, TE $=30 \mathrm{~ms}$, flip angle $=90^{\circ}, \mathrm{FOV}=240 \mathrm{~mm}, 36$ slices covering the whole brain, slice thickness $=3.8 \mathrm{~mm}$, in-plane resolution $\left.=3.8 \mathrm{~mm}^{2}\right)$. Steady state magnetization was assumed after five frames $(\sim 10 \mathrm{~s})$. The parameters for this acquisition have been optimized (e.g., oblique acquisition) to reduce susceptibility artifact, which causes signal drop out in orbitofrontal cortex. Three rest runs of 3.5-min duration obtained for all children. Two rest runs of 5-min duration were obtained for all adolescents and adults. During rest periods, subjects were verbally instructed to continue to stay still, view a 
Table 1 | Subject characteristics.

\begin{tabular}{|c|c|c|c|c|c|c|}
\hline & \multicolumn{2}{|c|}{ Adults } & \multicolumn{2}{|c|}{ Adolescents } & \multicolumn{2}{|c|}{ Children } \\
\hline & Mean & Std. Dev. & Mean & Std. Dev. & Mean & Std. Dev. \\
\hline Age & 25.33 & 3.85 & 13.02 & 1.37 & 8.57 & 0.67 \\
\hline Mvmt. (rms) & 0.36 & 0.40 & 0.41 & 0.21 & 0.34 & 0.33 \\
\hline Gender & $\%$ & $N$ & $\%$ & $\mathrm{~N}$ & $\%$ & $\mathrm{~N}$ \\
\hline Male & 50 & 7 & 61.90 & 13 & 29.4 & 5 \\
\hline Female & 50 & 7 & 38.10 & 8 & 70.6 & 12 \\
\hline
\end{tabular}

cross in the middle of the screen, and be sure to stay awake. The stimulus display consisted of the standard fixation-cross in the center of the stimulus field.

Functional images were processed to reduce artifacts (Miezin et al., 2000). These steps included: (i) removal of a central spike caused by MR signal offset, (ii) correction of odd vs. even slice intensity differences attributable to interleaved acquisition without gaps, (iii) correction for head movement within and across runs, and (iv) within run intensity normalization to a whole brain mode value of 1000. Atlas transformation of the functional data was computed for each individual via the MP-RAGE scan. The fMRI data then were resampled ( $3 \mathrm{~mm}$ cubic voxels) in Talairach atlas space (Talairach and Tournoux, 1988) as defined by the spatial normalization procedure (Lancaster et al., 1995). This resampling combined movement correction and atlas transformation in one interpolation. All subsequent operations were performed on the atlas-transformed volumetric time series. For presentation purposes, voxel boundaries were interpolated to $1 \mathrm{~mm}^{3}$ voxels and displayed using CARET software (Van Essen et al., 2001).

Participant head motion was measured and corrected using rigid body translation and rotation. Summary statistics were calculated as root mean square (RMS) values for translation and rotation about the $x, y$, and $z$-axes. Total RMS values were calculated on a run-by-run basis for each participant. BOLD runs with excessive movement ( $>2 \mathrm{~mm}$ RMS) were excluded from further analysis. Movement was relatively low in all groups (see Table $\mathbf{1}$ ).

\section{FUNCTIONAL CONNECTIVITY PRE-PROCESSING}

The functional data were additionally pre-processed in two ways for two separate analysis strategies, as outlined below. In the first analysis (total (marginal) correlation - see below) pre-processing was carried out as previously described (Fox et al., 2005; Fair et al., 2007a,b, 2008, 2009) to reduce spurious variance unlikely to reflect neuronal activity (Fox and Raichle, 2007). These steps included: (i) a temporal bandpass filter $(0.009 \mathrm{~Hz}<f<0.08 \mathrm{~Hz}$ ), (ii) regression of six parameters obtained by rigid body head motion correction, (iii) regression of the whole brain signal averaged over the whole brain, (iv) regression of ventricular signal averaged from ventricular region of interest (ROI), and ( $\mathrm{v}$ ) regression of white matter signal averaged from a white matter ROI. Regression of first derivative terms for whole brain, ventricular, and white matter signals were also included in the correlation pre-processing. These pre-processing steps are, in part, intended to remove developmental changes in connectivity driven by changes in respiration and heart rate over age. Pre-processing in preparation for the second analysis (partial correlation - see below) was similar except that the whole brain signal was not included as a nuisance regressor. The correlation procedures associated with these two separate pre-processing strategies are described below (in Correlations between cortical subdivisions and thalamus).

\section{CORTICAL ROI DEFINITION}

Cortical ROI definition was as in Zhang et al. (2008). In short, the cortex in each hemisphere was partitioned into five subregions (Figure 1). The MP-RAGE image from a normal young adult volunteer (not included in this study) was segmented along the gray/white boundary and deformed to the population-average, landmark and surface-based (PALS)-B12 atlas (Van Essen, 2005) using SureFit and CARET software (Van Essen and Drury, 1997; Van Essen et al., 2001). Partition boundaries were manually drawn based on major sulcal landmarks, following work by Behrens et al. (2003). Five cortical ROIs were thus defined: (1) frontopolar and frontal cortex including the orbital surface and anterior cingulate; (2) motor and premotor cortex (Brodmann areas 6 and 4 - excluding adjacent portions of cingulate cortex); (3) somatosensory cortex (Brodmann areas 3, $1,2,5$, and parts of 40); (4) parietal and occipital cortex including posterior cingulate and lingual gyrus; (5) temporal cortex including the lateral surface, temporal pole, and parahippocampal areas (Figure 1). For details see (Zhang et al., 2008, 2009).

\section{CORRELATIONS BETWEEN CORTICAL SUBDIVISIONS AND THALAMUS}

Resting state (fixation) data from 52 subjects ( 17 aged 7-9; 21 aged 11-16; 14 aged 19-32) were included in the analyses. The adolescent age range was chosen for two reasons. First, while there are several ways of defining adolescence, we chose an age-range that best encompasses the peripubertal years - a time of dynamic behavioral and neural maturation (Paus, 2005). By age 11, many children have initiated early pubertal processes (especially among females), and by age 16 , most youth have attained pubertal maturation (Kreipe, 1992). The second consideration regarded our prior connectivity results, which have shown significant transitions in connectivity between similar age groups (e.g., see Fair et al., 2007a). All participants contributed between $420-630$ s of resting-state data. The data were first analyzed with a total correlation procedure, which included whole brain signal regression in the initial pre-processing steps (see Functional Connectivity Pre-processing). In this case, for the five cortical subdivisions, an average resting state timeseries was extracted and correlated $(r)$ with all voxels of the thalamus separately and for each individual. Shared variance among the five cortical subdivisions is accounted for in this instance with the initial whole brain signal regression. This procedure is similar to the total correlation procedure used in Zhang et al. (2008). In the second analysis, whole brain signal regression was not used in the initial pre-processing. Rather, shared variance was accounted for by partial correlation, wherein the correlation between a cortical ROI and the thalamus was computed after covarying out the other four cortical regions. Total correlation yielded slightly less specificity but more uniformity across subjects in comparison to partial correlation. In both analyses, to calculate statistical significance within each age group, we first applied Fischer's $z$ transformation to the correlation coefficients $(r)$ to improve normality. From here, these values were converted to $Z$ scores by dividing by the square root of the variance within each group, as in Fox et al. (2005). $Z$-score maps were then 
combined across subjects using a fixed effects analysis. Results presented here are restricted to the thalamus, whose boundaries were created by manual tracing of the atlas template. For the 'winner take all' strategy (see Figure 3), the cortical subdivision that correlated most strongly for any given voxel was assigned the designated color for the 'winning' cortical subdivision.

To test significant change over development, direct comparisons between the two end point groups, children and adults, were performed. This between-group end point approach, as opposed to using the entire sample, has been shown to be more robust to potential non-linear changes (Fair et al., 2006). For such direct comparisons, we performed two-sample, two-tailed $t$-tests (random effects analysis assuming unequal variance; $p \leq 0.05$ ) for each cortical subdivision applied to Fischer $z$-transformed $r$ values. For the voxelwise, fixed effects maps, thresholding based on Monte Carlo simulation was implemented (Forman et al., 1995). To obtain multiple comparisons corrected, $p<0.05$ voxel clusters, a threshold of 35 contiguous voxels with a $Z$-value $>2.5$ was used. Maps showing statistically significant changes with age were uncorrected (as few voxels passed our stringent correction), and displayed with a threshold of $Z>2$.

\section{RESULTS}

\section{FUNCTIONAL CONNECTIVITY OF CORTICAL SUBDIVISIONS WITHIN THE THALAMUS IN ADULTS SHOW A SPATIAL ORGANIZATION IN AGREEMENT WITH KNOWN THALAMIC NUCLEAR GROUPING}

Replicating prior reports, (Zhang et al., 2008, 2009), in our adult sample, correlations between the thalamus and each cortical subdivision were distinct, with substantial correspondence with known axonal connectivity with thalamic nuclei in primates (Jones, 2007; Morel, 2007). Specifically, the parietal-occipital cortical subdivision showed strong correlations with the lateral and posterior portions of the thalamus. This portion of the thalamus and dorsal brain stem are comprised of lateral pulvinar, lateral geniculate, and superior colliculus, which contain combinations of afferent input, and projections, to parietal occipital association areas and primary visual cortex (Lock et al., 2003; Jones, 2007) (see Figure 2 - row 1, column 2; Figure S1 in Supplementary Material). The temporal cortical ROI correlated strongly with medial, inferior, and posterior portions of the thalamus. This segment of the thalamus and dorsal brainstem presumptively corresponds to medial pulvinar, inferior/ superior colliculi, medial geniculate, and medial dorsal nucleus (Webster et al., 1995; Jones, 2007), which have combinations of inputs from, and projections to, temporal cortex (see Figure 2-row 1, column 5; Figure S2 in Supplementary Material). The prefrontal cortical subdivision showed strong interactions with dorsal, medial, and anterior portions of the thalamus. This thalamic area contains medial dorsal and the anterior nuclear groups, with inputs and outputs to cingulate and prefrontal portions of the cortex (Jones, 2007) (see Figure 2 - row 1, column 1; Figure S3 in Supplementary Material). Somatosensory cortical areas strongly correlated with ventral, lateral, and posterior thalamic regions, which presumptively correspond to ventral posteriolateral and posteriomedial nuclei (Jones, 2007); see Figure 2, row 1, column 4, and Figure S4 in Supplementary Material. Finally, the motor cortex subdivision correlated strongly with lateral and ventral thalamic areas that presumptively correspond to ventral lateral and ventral anterior nuclei (see Figure 2-row 1, column 3; Figure S5 in Supplementary
Material). As can be seen in Figure 3, these findings are perhaps most clearly evident in 'winner take all' displays (Zhang et al., 2008, 2009); also see Figure S6 in Supplementary Material).

\section{FUNCTIONAL CONNECTIVITY OF CORTICAL SUBDIVISIONS WITH THE THALAMUS CHANGES SUBSTANTIALLY OVER DEVELOPMENT}

Although thalamic nuclear groups are defined early in development (Jones, 2007), we saw substantial differences in connectivity patterns between our younger participants and adults. Figure 2 (rows 1-3) suggests a transitional change from childhood, through adolescence, to adulthood for thalamo-frontal and thalamo-temporal interactions. Specifically, frontal lobe correlations are weak in childhood and appear to strengthen by adulthood. Temporal lobe correlations with the thalamus, however, are much stronger in children, and weaken progressively in adolescence and adulthood. This finding was obtained both by total correlation (Figure 2) and partial correlation analyses (Figure S7 in Supplementary Material). The finding also held true when looking at raw correlation values $(r)$, rather than $z$ statistics (Figure S8 in Supplementary Material).

This particular finding (i.e., increased thalamo-frontal interactions and decreased thalamo-temporal interactions over age) is clearly seen in the 'winner take all' displays. In adolescents (Figure 3), the picture was slightly modified from what was seen in the adult group. Along the midline bilaterally, the thalamo-temporal correlations encompassed a greater portion of anterior and midline thalamus, while frontal lobe correlations encompassed much less of the anterior portions of the thalamus. This trend, in which frontal (and to a lesser extent, motor) correlations give way to temporal correlations, was observed to an even greater extent in the youngest age group. In children, thalamo-temporal correlations were stronger and more widespread - not only encroaching on areas occupied by thalamo-frontal correlations in adults, but also parts of the thalamus functionally connected with motor/premotor, somatosensory, and occipital/parietal areas. In contrast, the spatial extent of the thalamo-frontal interaction was minimal in children and somewhat stronger in the adolescent group although, still limited relative to the adults. This dynamic can also be observed in Movie S1-S4 in Supplementary Material. Again, Figure S9 in Supplementary Material shows these findings were largely unchanged when using partial correlations. (Our child and adolescent groups had a slight excess of females and males, respectively. Accordingly, we repeated our analysis using equal numbers of males and females in each group. These findings, shown in Figure S10 in Supplementary Material, suggest that gender had minimal effect on the overall patterns observed here.)

Direct statistical comparisons between children and adults confirmed the qualitative observations for the frontal-thalamic and temporal-thalamic interactions. Thalamo-cortical interactions observed with somatosensory cortex were qualitatively similar, but showed statistical differences between groups of both increased (lateral/inferior) and decreased (medial/dorsal) connectivity with specific parts of the thalamus (Figure 4). Similar findings were observed for motor-premotor cortex. Correlations with the occipital/parietal ROI appeared qualitatively unchanged across age groups. This observation was also confirmed with the direct statistical comparisons (Figure 4). The direct comparisons between children and adults using partial correlations (without whole brain 


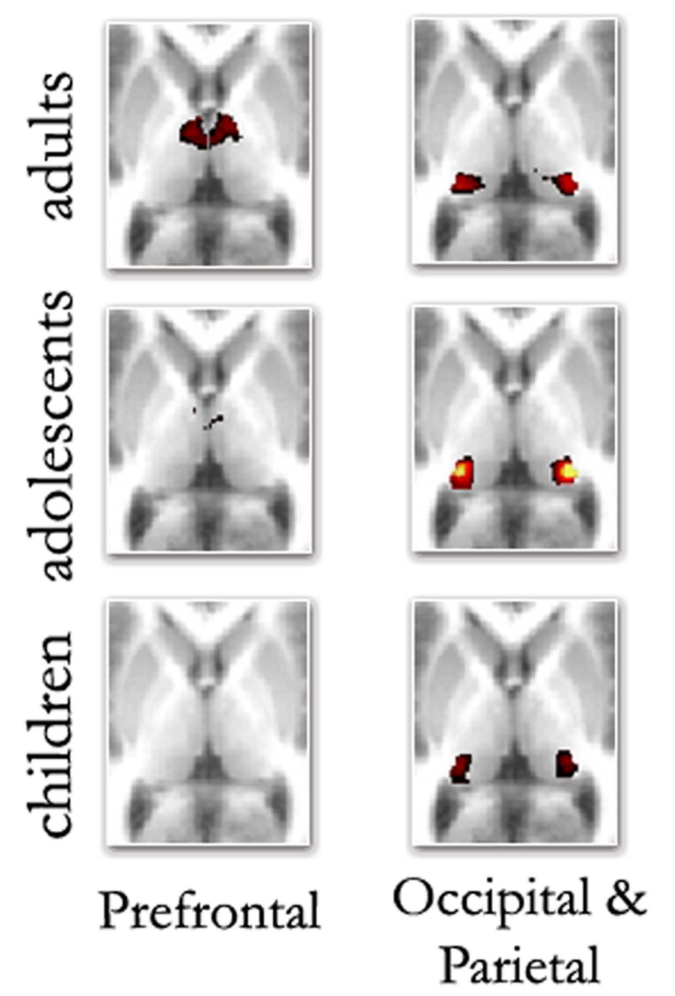

FIGURE 2 | Fixed effects functional mapping of the thalamus for each cortical ROI, in each age group (Children, Adolescents, Adults). This qualitative comparison appears to show substantial change over development regarding the thalamo-cortical functional interactions. The functional neuroanatomy of the adult group is quite similar to previous publications
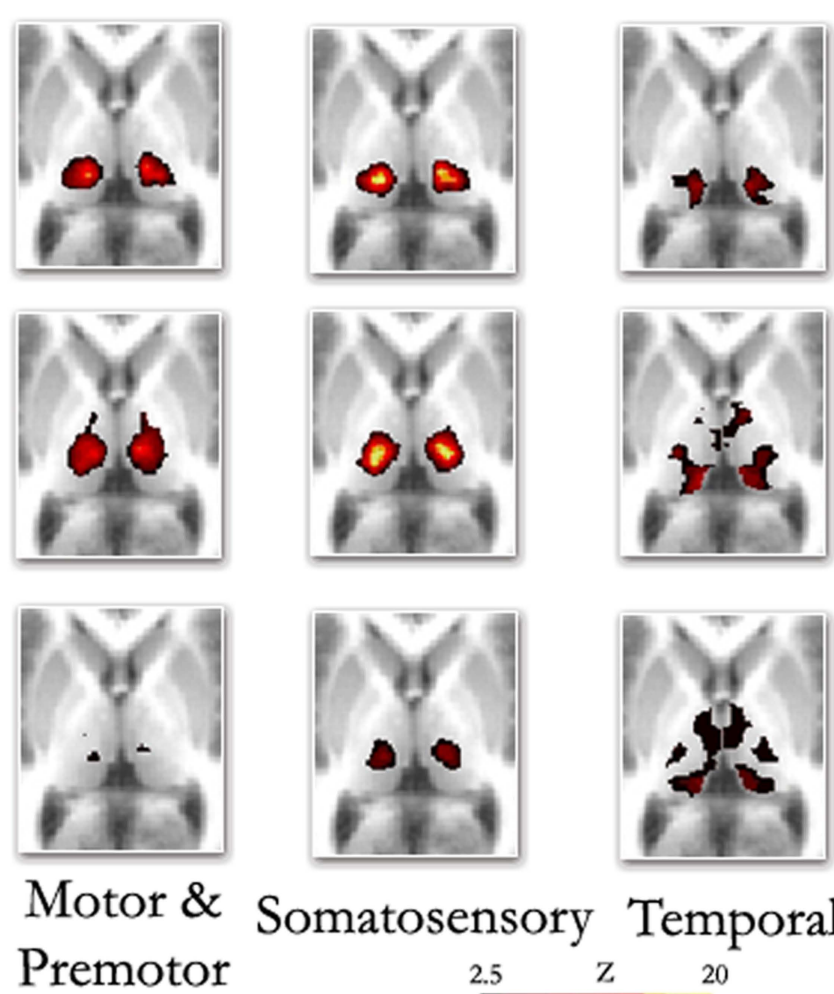
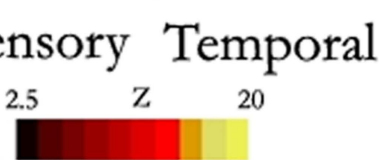

signal regression - Figure S11 in Supplementary Material) were slightly different in that statistical differences observed between somatosensory and motor-premotor ROIs appeared weaker.

\section{DISCUSSION}

In this report we replicate prior findings that demonstrate spatially distinct BOLD correlations between the thalamus and specific cortical subdivisions that are in substantial correspondence with known thalamic connectivity in primates (Jones, 2007; Morel, 2007). We also saw significant development in thalamo-cortical correlations over maturation via rs-fcMRI. Specifically, we showed a progressive strengthening of functional connectivity of the frontal cortex with dorsal/anterior subdivisions of the thalamus. We also saw a systematic weakening of temporal lobe connectivity with ventral/ midline/posterior subdivisions of the thalamus. Premotor-motor and somatosensory cortical subdivisions also showed increased connectivity in lateral/inferior portions of the thalamus and decreased connectivity in medial/dorsal portions of the thalamus. Occipital-parietal correlations with the thalamus were relatively stable over our samples.

Of note, considering the nature of the BOLD response, it is conceivable that developmental differences in the hemodynamic response could affect our results (D'Esposito et al., 2003). We
(Zhang et al., 2008, 2009). The most prominent differences between age groups shows frontal lobe interactions that are weaker in children and appear to increase over age. In contrast, temporal lobe interactions are much stronger in children, but weaken progressively in adolescents and adults: Transverse $Z=+8$ feel this is unlikely considering reports suggesting that changes observed over development with fMRI are not the product of changes in hemodynamic response mechanisms over age (Kang et al., 2003; Wenger et al., 2004). Similarly, we also note that our observations could be affected by physiologic noise such as respirations and heart rate, but believe this is also unlikely for two reasons. First, most of these nuisance signals are likely removed with our band-pass filter and/or the removal of shared variance via partial correlations or whole brain signal regression. Second, the observations in this report (and elsewhere, Fair et al., 2007a, 2009; Kelly et al., 2009; Supekar et al., 2009) show age-related changes over development that occur in both directions (i.e., strengthen and weaken). It would be difficult to explain how a difference in heart rate or respiration could account for these opposing dynamics. With that said, it would be beneficial for future reports to include these additional physiologic noise parameters as regressors into the processing strategy of rs-fcMRI; however, it will be equally interesting in future reports to determine how brain oscillations might actually drive cardiac and respiratory fluctuations. Assuming that the age-related alterations described here represent true change in functional connections, the question then becomes: What are the neurobiological underpinnings of that change? 


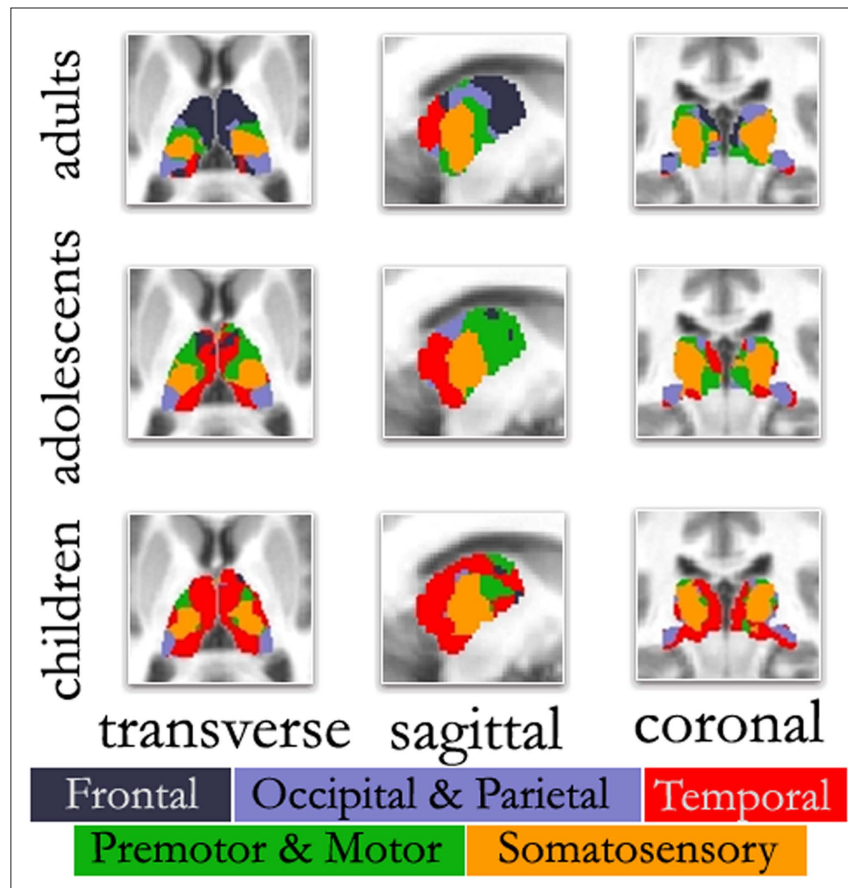

FIGURE 3 |'Winner take all' displays across age. As previously observed, the color-coded thalamus based on the winner take all strategy in adults shows a functional organization that is remarkably similar to known nuclear groupings in the primate thalamus. However, the picture is different in adolescents and children. In agreement with Figure 2, in both adolescents and to a greater extent children, thalamo-temporal interactions encompass a greater portion of anterior and midline thalamus, while the frontal lobe interactions encompass much less of the anterior portions of the thalamus. In children, thalamo-temporal interactions not only encroach on areas that, in adults, are dominated by thalamo-frontal interactions, but also impinge on thalamic zones that later become functionally more connected with motor/ premotor, somatosensory, and occipical/parietal cortex (also see Movies in Supplementary Material); Transverse $Z=+8$, Sagittal $X=-12$, Coronal $Y=-27$.

\section{CHANGES IN THE FUNCTIONAL RELATIONSHIPS OF CORTICAL STRUCTURES WITH THE THALAMUS MAY, IN PART, REFLECT CHANGES IN THE UNDERLYING NEURAL SUBSTRATE}

There are multiple sources of developmental change that may account for the changes in functional connectivity observed from the child to adult thalamus reported here. Based on comparisons of this method with similar methods using DTI in adults (Zhang et al., 2009), it is clear that some of the functional relationships seen here are related to large fiber-tracts connecting the cortex with specific subdivisions of the thalamus. This is not to say that nuclear groupings are not fully developed early in development (at birth thalamic nuclei are known to be composed of connectional and functionally distinct cell types; Jones, 2007). Nor is this to say that large fiber bundles are growing or being eliminated over the age span studied here; these are in place by $\sim 9$ months of age (Conel, 1939-1963). However, there does continue to be significant refinement in connectional anatomy that occurs well into young adulthood.

For example, axonal retraction and the elimination of axon collaterals continues throughout development and aging (O'Leary, 1989; Luo and O'Leary, 2005). Following a proliferation of synapses early in development, there is a protracted period of synaptic pruning that reaches adult levels in the late second decade of life (Huttenlocher, 1979; Huttenlocher et al., 1982; Elman et al., 1996; Huttenlocher and Dabholkar, 1997; Casey et al., 2005; Jones, 2007). Importantly, these regressive processes are not random. They are selective, reduce connectivity between specific regions, and occur in both cortical and subcortical structures (Ebbesson, 1980; Greenough et al., 1987; Luo and O'Leary, 2005; Jones, 2007).

Such phenomena may account for some of the findings shown here. For example, work conducted by Webster et al. (1995) in developing macaques provides a compelling parallel with regard to weakening thalamo-temporal connectivity over age. In this study, the authors compared the subcortical connections of inferior temporal cortex (Areas TE and TEO) in infant vs. adult monkeys. While the connectional anatomy was similar in infant and adult monkeys, there was significant refinement of the subcortical connections. Specifically, while projections from TE (and to a more limited extent TEO) to the nucleus medial dorsalis were present in infants and adults, they were more widespread in infants and significantly reduced in the adult animals. The same was true for connections from area TE projecting to the superior colliculus. Similar changes potentially occur in medial and polar aspects of the temporal lobe (Russchen et al., 1987). Importantly, both frontal and temporal cortical subdivisions share anatomical connections to the nucleus medial dorsalis (Webster et al., 1995; Jones, 2007), which is consistent with the thalamo-temporal and thalamo-frontal connectivity seen here (Figure 3). Hence, the refinement of inferior temporal lobe projections directly to nucleus medial dorsalis, may, in part, account for the reduced thalamo-temporal connectivity observed during development.

The reduced connectivity associated with somatosensory and motor-premotor cortical subdivisions is also likely related to similar phenomena as the temporal lobe. For example, in mice, relay neurons of the ventral posterior medial nucleus undergo significant dendritic refinement over age, with more than $300 \%$ reduction in the extent of their dendritic fields from age P6 to adulthood (Brown et al., 1995; Zantua et al., 1996; Jones, 2007). Work by Dennis O'Leary and colleagues (Luo and O'Leary, 2005) has shown in rodents, that cortical layer $\mathrm{V}$ neurons, after early extensive interstitial branching, acquire functionally appropriate connections through selective elimination, dictated by the cortical area in which the neuron is located. For example, in newborns, motor and visual neurons project to common targets in the brainstem and spinal cord. During maturation, functionally appropriate connections are acquired through selective axon elimination, determined by the cortical area (i.e., motor or visual) in which the neuron is located.

In addition to a reduction in thalamo-temporal correlations, we also demonstrated increased thalamo-frontal correlations across development. One commonly cited contributor to increased connectivity between regions is myelination. Indeed, myelination has been shown to be closely related to rs-fcMRI measures (Hagmann et al., 2008; Greicius et al., 2009) (although this has not yet been examined in children). Myelination increases at least through young adulthood. It proceeds from primary sensory and motor regions to association areas (Flechsig, 1920; 


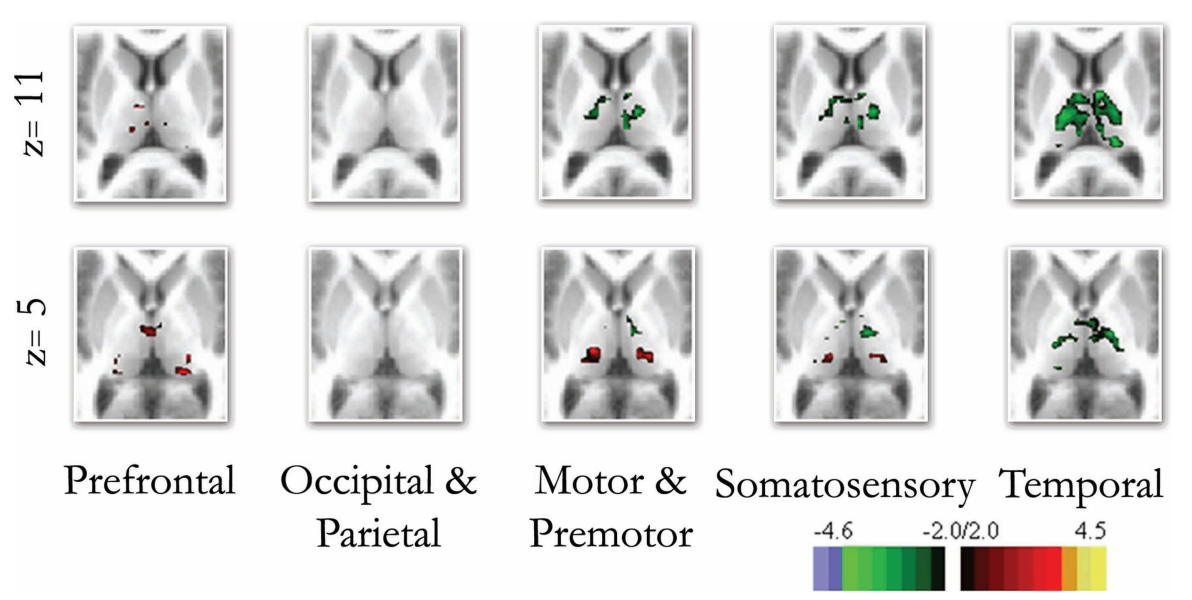

FIGURE 4 | Direct comparison between the end groups (i.e., children and adults). The direct comparison between children and adults confirmed many of the qualitative findings shown in Figure 3. Frontal connectivity with the thalamus increases with age, and Temporal connectivity with the thalamus weakens with age. Differences in connectivity between children and adults with premotor/motor and somatosensory cortex were also revealed with the direct comparisons, while the occipical/parietal $\mathrm{ROI}$ showed very little difference between the groups.
Brody et al., 1987; Paus et al., 2001; Sowell et al., 2002), roughly following the hierarchical organization introduced by Felleman and Van Essen (1991). As such, increased signal propagation, through the maturation of the myelin sheath, is a likely contributor to the increased interaction between the frontal cortex and subregions of the thalamus, particularly those in the anterior and medial dorsal portion (Luna and Sweeney, 2004; Fair et al., 2007a, 2008; Kelly et al., 2009).

\section{CHANGES IN THE FUNCTIONAL RELATIONSHIPS OF CORTICAL STRUCTURES WITH THE THALAMUS LIKELY REFLECT CHANGES BEYOND THE MATURING NEURAL SUBSTRATE}

Changes in cortical-subcortical dynamics, particularly with the frontal cortex, are likely not solely due to changes in the underlying neural substrate (Honey et al., 2007; Fair et al., 2009; Lewis et al., 2009). It is now apparent that the connectivity signal measured via rs-fcMRI is not a pure representation of monosynaptic anatomical connectivity (Vincent et al., 2007; Hagmann et al., 2008; Zhang et al., 2008), and thus, other explanations must be considered. For example, modeling work has shown that complex spatial and temporal patterns of synchronous activity can develop over time in the absence of external input and without changes in the neuroanatomy (Honey et al., 2007). Other work by Galinanes et al. (2009) has shown, in mice, that subcortical neurons in the striatum are more temporally tuned to frontal cortical rhythms in adults than they are in infancy (i.e., they are more strongly functionally connected - albeit in different frequency ranges than examined here). Importantly, Galinanes found that these changes in functional properties are unlikely to be secondary to direct development of anatomical changes per se, but rather the modulation of functional properties through the maturation of the dopaminergic system. While this work targeted the striatum, similar phenomena could be occurring indirectly (or directly) in the thalamus without direct changes in the gross neuroanatomy, and independently of myelination.
These types of functional changes identified by Galinanes and colleagues highlight an important aspect regarding thalamic organization and the developmental changes observed in this report. As put by Sherman and Guillery (2006), 'It is important to distinguish the functional input that carries the messages for transmission to the cortex, the driver, from the many other inputs, the modulators, which can modify the way in which the message is transmitted...' The circuitry of the thalamus is complex. Its function is determined not only by the driver connectional anatomy to the cortex, but also modulators (interneurons, other subcortical inputs, various neurotransmitter systems) (Sherman and Guillery, 2006; Jones, 2007). Indeed, throughout the thalamus, driver synapses to relay cells encompass only a fraction of the total number synapses ( 2-10\%) (Sherman and Guillery, 2006). To the contrary, modulatory synapses to relay cells account for over $90 \%$ of synaptic contacts with relay neurons. Importantly, many properties related to modulatory action continue to develop postnatally (Jones, 2007). Hence, it is likely that increases and decreases in thalamo-cortical connectivity seen here over age are influenced by maturation of modulatory systems (Jones, 2007).

\section{FUTURE DIRECTIONS}

In this report, we showed dynamic maturing functional interactions between the thalamus and cortex. The gross partitioning of cortical regions, as used in the present study, is adequate to produce connectivity maps with thalamic nuclei that are consistent with known structural connectivity, and is promising. In addition, there appears to be little difference in results obtained by partial vs. total correlation analysis. However, it is important to note that while the currently applied methods appear well suited for identifying distinct subcortical structures in adults, they do not provide the same specificity in children. This difference likely reflects functional and anatomic maturational mechanisms. Alternative rsfcMRI techniques may be better suited for identifying nuclear 
groupings specific to children and adolescents (e.g., Cohen et al., 2008; Margulies et al., 2009). Other complementary connectivity methods may additionally assist in differentiating changes across development related to extent versus strength or magnitude in connectivity. For example, as noted in the Introduction section, anatomically distinct nuclear groups and large fiber tracts are well formed within the thalamus early in development (Jones, 2007). As such, anatomically based methods that utilize large fiber bundles (see Behrens et al., 2003; Asato et al., 2010, as opposed to functionally based methods, may prove successful in further differentiating nuclear groupings and extent of connectivity in children.

In future work it will also be necessary to observe how changes in thalamo-cortical functional connectivity relate to developmental changes in behavior. It is likely that the cortical-subcortical interactions observed here will correspond to a shift from reflexive, stimulus-bound behavior in childhood, to the goaldirected, self-organized, and more flexible functioning in young adulthood (Stuss, 1992) - a distinct hypothesis that can be tested. For example, recent theories suggest that, during childhood and early adolescence, goal-directed behavior is governed by principles of approach and avoidance, with regulation and balance of this system developing across adolescence and into adulthood (Ernst et al., 2009). While early approach and avoidance is thought to

\section{REFERENCES}

Alexander, G. E., and Crutcher, M. D. (1990). Basal ganglia-thalamocortical circuits: parallel substrates for motor,oculomotor,prefrontal and limbic functions. Prog. Brain Res. 85, 119-146.

Andrews-Hanna,J.R.,Snyder,A.Z., Vincent, J. L., Lustig, C., Head, D., Raichle, M. E., and Buckner, R. L. (2007). Disruption of large-scale brain systems in advanced aging. Neuron 56, 924-935.

Asato, M. R., Terwilliger, R., Woo, J., and Luna, B. (2010). White matter developmentinadolescence:aDTIstudy. Cereb. Cortex. doi:10.1093/cercor/bhp282.

Behrens, T. E., Johansen-Berg, H., Woolrich, M. W., Smith, S. M., Wheeler-Kingshott, C.A., Boulby, P.A., Barker, G. J., Sillery, E. L., Sheehan, K., Ciccarelli, O., Thompson, A. J., Brady, J. M., and Matthews, P. M. (2003). Non-invasive mapping of connections between human thalamus and cortex using diffusion imaging. Nat. Neurosci. 6, 750-757.

Biswal, B., Yetkin, F. Z., Haughton, V. M. and Hyde, J.S. (1995). Functional connectivity in the motor cortex of resting human brain using echo-planar MRI. Magn. Reson. Med. 34, 537-541.

Brody, B. A., Kinney, H. C., Kloman, A. S., and Gilles, F. H. (1987). Sequence of central nervous system myelination in human infancy. I. An autopsy study of myelination. J. Neuropathol. Exp. Neurol. 46, 283-301.
Brown, K. E., Arends, J. J., Wasserstrom, Woolsey, T.A. (1995). Developmental transformation of dendritic arbors in mouse whisker thalamus. Brain Res. Dev. Brain Res. 86, 335-339.

Bunge, S. A., Dudukovic, N. M., Thomason, M. E., Vaidya, C. J., and Gabrieli, J. D. (2002). Immature frontal lobe contributions to cognitive control in children: evidence from fMRI. Neuron 33, 301-311.

Casey, B. J., Jones, R. M., and Hare, T. A. (2008). The adolescent brain. Ann. N. Y. Acad. Sci. 1124, 111-126.

Casey, B. J., Tottenham, N., Liston, C., and Durston, S. (2005). Imaging the develabout cognitive development? Trends Cogn. Sci. 9, 104-110.

Chambers, R. A., Taylor, J. R., and Potenza, M.N.(2003).Developmental neurocircuitry of motivation in adolescence: a critical period of addiction vulnerability. Am. J. Psychiatry 160, 1041-1052.

Church, J.A., Fair, D.A., Dosenbach, N.U., Cohen, A. L., Miezin, F. M., Petersen, S. E., and Schlaggar, B. L. (2009). Control networks in paediatric Tourette syndrome show immature and anomalous patterns of functional connectivity. Brain 132(Pt 1), 225-238.

Cohen, A. L., Fair, D. A., Dosenbach, N. U., Miezin, F. M., Dierker, D., Van Essen, D. C., Schlaggar, B. L., and Petersen, S. E. (2008). Defining functional areas in individual human brains using S. P., Zantua, J. B., Jacquin, M. F., and oping brain: what have we learned

be subserved by subcortical and limbic brain regions (consistent with greater thalamo-temporal interactions in children), emerging control of these affective and appetitive behaviors (among others - see Bunge et al., 2002) occurs in concert with the maturation of subcortical to prefrontal cortex interactions (consistent with emerging increased thalamo-frontal interactions shown across our sample) (Chambers et al., 2003; Casey et al., 2008). Along the same lines, identifying how these cortical-subcortical interactions relate to models of neuropsychiatric disorders will also be of interest in future work.

\section{ACKNOWLEDGMENTS}

Research was supported by the Oregon Clinical and Translational Research Institute (Fair), Medical Research Foundation (Fair), UNCF Merck postdoctoral fellowship (Fair), Ford Foundation (Fair), R01 MH59105 (Nigg), NS06833 (Raichle), K08 NS52147 (Nagel), Portland Alcohol Research Center (P60 AA010760 Nagel), and the OHSU Neuropsychiatric Institute (Nigg).

\section{SUPPLEMENTARY MATERIAL}

The Supplementary Material for this article can be found online at http://www.frontiersin.org/systemsneuroscience/paper/10.3389/ fnsys.2010.00010/

resting functional connectivity MRI. Neuroimage 41, 45-57.

Conel, J. L. (1939-1963). The Postnatal Development of the Human Cerebral Cortex. Cambridge, MA: Harvard University Press.

D’Esposito, M., Deouell, L. Y., and Gazzaley, A. (2003). Alterations in the BOLD fMRI signal with ageing and disease: a challenge for neuroimaging. Nat. Rev. Neurosci. 4, 863-872.

Dosenbach, N. U., Fair, D. A., Miezin, F. M., Cohen, A. L., Wenger, K. K., Dosenbach, R. A. T., Fox, M. D. Snyder, A. Z., Vincent, J. L., Raichle, M. E., Schlaggar, B. L., and Petersen, S. E. (2007). Distinct brain networks for adaptive and stable task control in humans. PNAS 104, 11073-11078.

Ebbesson, S. O. (1980). The parcellation theory and its relation to interspecific variability in brain organization, evolutionary and ontogenetic development, and neuronal plasticity. Cell Tissue Res. 213, 179-212.

Elman, J. L., Bates, E. A., Johnson, M. H., and Karmiloff-Smith, A. (1996). Rethinking Innateness: A Connectionist Perspective on Development. Cambridge, MA: The MIT Press.

Ernst, M., Romeo, R. D., and Andersen, S. L. (2009). Neurobiology of the development of motivated behaviors in adolescence: a window into a neural systems model. Pharmacol. Biochem. Behav. 93, 199-211.
Fair, D. A., Brown, T. T., Petersen, S.E., and Schlaggar, B. L. (2006). A comparison of ANOVA and correlation methods for investigating cognitive development with fMRI. Dev. Neuropsychol. 30, 531-546.

Fair, D. A., Cohen, A. L., Dosenbach, N. U., Church, J. A., Miezin, F. M. Barch, D. M., Raichle, M. E., Petersen, S. E., and Schlaggar, B. L. (2008). The maturing architecture of the brain's default network. PNAS 105 4028-4032.

Fair, D. A., Cohen, A. L., Power, J. D., Dosenbach, N. U., Church, J. A. Miezin, F. M., Schlaggar, B. L., and Petersen, S.E. (2009). Functional brain networks develop from a "local to distributed” organization. PLoS Comput. Biol. 5, e1000381. doi: 10.1371/journal. pcbi.1000381.

Fair, D. A., Dosenbach, N. U. F., Church, J. A., Cohen, A. L., Brahmbhatt, S., Miezin, F. M., Barch, D. M., Raichle, M. E., Petersen, S. E., and Schlaggar, B. L. (2007a). Development of distinct control networks through segregation and integration. PNAS 104, 13507-13512.

Fair, D. A., Schlaggar, B. L., Cohen, A. L., Miezin, F. M., Dosenbach, N. U., Wenger, K. K., Fox, M. D., Snyder, A. Z., Raichle, M. E., and Petersen, S. E. (2007b). A method for using blocked and event-related fMRI data to study "resting state" functional connectivity. Neuroimage 35, 396-405. 
Felleman, D. J., and Van Essen, D. C. (1991). Distributed hierarchical processing in the primate cerebral cortex. Cereb. Cortex 1, 1-47.

Flechsig, P. E. (1920). Anatomie des Menschlichen Gehirn und Ruckenmarks, auf Myelogenetischer Grundlage. Leipzig: G. Thieme.

Forman, S. D., Cohen, J.D., Fitzgerald, M., Eddy, W. F., Mintun, M. A., and Noll, D. C. (1995). Improved assessment of significant activation in functional magnetic resonance imaging (fMRI): use of a cluster-size threshold. Magn. Reson. Med. 33, 636-647.

Fox, M. D., Corbetta, M., Snyder, A. Z., Vincent, J. L., and Raichle, M. E. (2006). Spontaneous neuronal activity distinguishes human dorsal and ventral attention systems. PNAS 103, 10046-10051.

Fox, M. D., and Raichle, M. E. (2007). Spontaneous fluctuations in brain activity observed with functional magnetic resonance imaging. Nat. Rev. Neurosci. 8, 700-711.

Fox, M. D., Snyder, A. Z., Vincent, J. L., Corbetta, M., Van Essen, D. C., and Raichle, M. E. (2005). The human brain is intrinsically organized into dynamic, anticorrelated functional networks. PNAS 102, 9673-9678.

Fransson, P., Skiold, B., Horsch, S., Nordell, A., Blennow, M., Lagercrantz, H., and Aden, U. (2007). Resting-state networks in the infant brain. PNAS 104, 15531-15536.

Friston, K. J., Frith, C. D., and Frackowiak, R. S. J. (1993). Time-dependent changes in effective connectivity measured with PET. Hum. Brain Mapp. 1, 69-80.

Galinanes, G. L., Taravini, I. R., and Murer, M. G. (2009). Dopamine-dependent periadolescent maturation of corticostriatal functional connectivity in mouse. J. Neurosci. 29, 2496-2509.

Giedd, J.N., Blumenthal, J., Jeffries, N. O., Castellanos, F. X., Liu, H., Zijdenbos, A., Paus, T., Evans, A.C., and Rapoport, J.L. (1999). Brain development during childhood and adolescence: a longitudinal MRI study. Nat. Neurosci. 2, 861-863.

Greenough, W. T., Black, J. E., and Wallace, C. S. (1987). Experience and brain development. Child Dev. 58, 539-559.

Greicius, M. D., Flores, B. H., Menon, V., Glover, G. H., Solvason, H. B., Kenna, H., Reiss, A. L., and Schatzberg, A. F. (2007). Resting-state functional connectivity in major depression: abnormally increased contributions from subgenual cingulate cortex and thalamus. Biol. Psychiatry 62, 429-437.

Greicius, M. D., Supekar, K., Menon, V., and Dougherty, R. F. (2009). Resting- state functional connectivity reflects structural connectivity in the default mode network. Cereb. Cortex 19, 72-78.

Hagmann, P., Cammoun, L., Gigandet, X., Meuli, R., Honey, C. J., Wedeen, V. J., and Sporns, O. (2008). Mapping the structural core of human cerebral cortex. PLoS Biol. 6, e159. doi: 10.1371/ journal.pbio.0060159.

Hampson, M., Driesen, N. R., Skudlarski, P., Gore, J. C., and Constable, R. T. (2006). Brain connectivity related to working memory performance. J. Neurosci. 26, 13338-13343.

Honey, C., Kotter, R., Breakspear, M., and Sporns, O. (2007). Network structure of cerebral cortex shapes functional connectivity on multiple time scales. PNAS 104, 10240-10245.

Huttenlocher, P. R. (1979). Synaptic density in human frontal cortex - developmental changes and effects of aging. Brain Res. 163, 195-205.

Huttenlocher, P. R., and Dabholkar, A. S. (1997). Regional differences in synaptogenesis in human cerebral cortex. J. Comp. Neurol. 387, 167-178.

Huttenlocher, P. R., de Courten, C., Garey, L. J., and Van der Loos, H. (1982). Synaptogenesis in human visual cortex-evidence for synapse elimination during normal development. Neurosci. Lett. 33, 247-252.

Johansen-Berg, H., Behrens, T. E., Sillery, E., Ciccarelli, O., Thompson, A. J., Smith, S. M., and Matthews, P. M. (2005). Functional-anatomical validation and individual variation of diffusion tractography-based segmentation of the human thalamus. Cereb. Cortex $15,31-39$.

Jones, E. G. (2007). The Thalamus. Cambridge, UK: University Press.

Kang, H.-S. C., Burgund, E. D., Lugar, H. M., Petersen, S. E., and Schlaggar, B. L. (2003). Comparison of functional activation foci in children and adults using a common stereotactic space. Neuroimage 19, 16-28.

Kelly, A. M., Di Martino, A., Uddin, L. Q., Shehzad, Z., Gee, D. G., Reiss, P. T., Margulies, D. S., Castellanos, F. X., and Milham, M. P. (2009). Development of anterior cingulate functional connectivity from late childhood to early adulthood. Cereb. Cortex 19, 640-657.

Kreipe, R. (1992). "Normal somatic adolescent growth and development," in Textbook of Adolescent Medicine, ed. L. Bralow (Philadelphia: W.B. Sounders Company), 44-67.

Lancaster, J. L., Glass, T. G., Lankipalli, B. R., Downs, H., Mayberg, H., and Fox, P. T. (1995). A modality-independent approach to spatial normalization of tomographic images of the human brain. Hum. Brain Mapp. 3, 209-223.
Lee, L., Harrison, L. M., and Mechelli, A. (2003). A report of the functional connectivity workshop, Dusseldorf 2002. Neuroimage 19(Pt 1), 457-465.

Lewis, C. M., Baldassarre, A., Committeri, G., Romani, G. L., and Corbetta, M. (2009). Learning sculpts the spontaneous activity of the resting human brain. PNAS 106, 17558-17563.

Lock, T. M., Baizer, J. S., and Bender, D. B. (2003). Distribution of corticotectal cells in macaque. Exp. Brain Res. 151, 455-470.

Luna, B., and Sweeney, J. A. (2004). The emergence of collaborative brain function: FMRI studies of the development of response inhibition. Ann. N. Y.Acad. Sci. 1021, 296-309.

Luo, L., and O’Leary, D. D. (2005). Axon retraction and degeneration in development and disease. Annu. Rev. Neurosci. 28, 127-156.

Mai, J., Paxinos, G., and Voss, T. (2008). Atlas of the Human Brain. Amsterdam: Elsevier/Academic Press.

Margulies, D. S., Vincent, J. L., Kelly, C., Lohmann, G., Uddin, L. Q., Biswal, B. B., Villringer, A., Castellanos, F. X., Milham, M.P., and Petrides, M. (2009). Precuneus shares intrinsic functional architecture in humans and monkeys. PNAS 106, 20069-20074.

Miezin, F., Maccotta, L., Ollinger, J., Petersen, S., and Buckner, R. (2000). Characterizing the hemodynamic response: effects of presentation rate, sampling procedure, and the possibility of ordering brain activity based on relative timing. Neuroimage 11, 735-759.

Morel, A. (2007). Stereotactic Atlas of the Human Thalamus and Basal Ganglia. New York: Informa Healthcare.

Nieuwenhuys, R. (1988). The Human Central Nervous System: A Synopsis and Atlas. New York: Springer-Verlag.

O’Leary, D.D.M. (1989). Do cortical areas emerge from a protocortex? Trends Neurosci. 12, 400-406.

Paus, T. (2005). Mapping brain maturation and cognitive development during adolescence. Trends Cogn. Sci. (Regul. Ed.) 9, 60-68.

Paus, T., Collins, D. L., Evans, A. C., Leonard, G., Pike, B., and Zijdenbos, A. (2001). Maturation of white matter in the human brain: a review of magnetic resonance studies. Brain Res. Bull. 54, 255-266.

Russchen, F. T., Amaral, D. G., and Price, J. L. (1987). The afferent input to the magnocellular division of the mediodorsal thalamic nucleus in the monkey, Macaca fascicularis. J. Comp. Neurol. 256, 175-210.

Seeley, W. W., Menon, V., Schatzberg, A. F., Keller, J., Glover, G. H., Kenna, H., Reiss, A. L., and Greicius, M. D.
(2007). Dissociable intrinsic connectivity networks for salience processing and executive control. J. Neurosci. 27, 2349-2356.

Sherman, S. M., and Guillery, R. W. (2006). Exploring the Thalamus and Its Role in Cortical Function. Cambridge: MIT Press.

Sowell, E. R., Trauner, D. A., Gamst, A., and Jernigan, T.L. (2002). Development of cortical and subcortical brain structures in childhood and adolescence: a structural MRI study. Dev. Med. Child Neurol. 44, 4-16.

Stuss, D. T. (1992). Biological and psychological development of executive functions. Brain Cogn. 20, 8-23.

Supekar, K., Musen, M., and Menon, V. (2009). Development of large-scale functional brain networks in children. PLoS Biol. 7, e1000157. doi: 10.1371/ journal.pbio.1000157.

Talairach, J., and Tournoux, P. (1988). CoPlanar Stereotaxic Atlas of the Human Brain. New York: Thieme Medical Publishers, Inc.

Uddin, L. Q., Kelly, A. M., Biswal, B. B., Margulies, D. S., Shehzad, Z., Shaw, D., Ghaffari, M., Rotrosen, J., Adler, L. A., Castellanos, F. X., and Milham, M. P. (2008). Network homogeneity reveals decreased integrity of defaultmode network in ADHD. J. Neurosci. Methods 169, 249-254.

Van Essen, D. C. (2005). A populationaverage, landmark- and surface-based (PALS) atlas of human cerebral cortex. Neuroimage 28, 635-662.

Van Essen, D. C., Dickson, J., Harwell, J., Hanlon, D., Anderson, C. H., and Drury, H. A. (2001). An integrated software suite for surface-based analyses of cerebral cortex. J. Am. Med. Inform. Assoc.41, 1359-1378. Available at: http://brainmap.wustl.edu/caret.

Van Essen, D. C., and Drury, H. A. (1997). Structural and functional analyses of human cerebral cortex using a surface-based atlas. J. Neurosci. 17, 7079-7102.

Vincent, J. L., Patel, G. H., Fox, M. D., Snyder, A. Z., Baker, J. T., Van Essen, D. C., Zempel, J. M., Snyder, L. H., Corbetta, M., and Raichle, M. E. (2007). Intrinsic functional architecture in the anesthetized monkey brain. Nature 447, 46-47.

Webster, M. J., Bachevalier, J., and Ungerleider, L. G. (1995). Transient subcortical connections of inferior temporal areas TE and TEO in infant macaque monkeys. J. Comp. Neurol. 352, 213-226.

Wenger, K. K., Visscher, K. M., Miezin, F. M., Petersen, S. E., and Schlaggar, B. L. (2004). Comparison of sustained and transient activity in children and adults using a mixed blocked/event- 
related fMRI design. Neuroimage 22, 975-985.

Zantua, J. B., Wasserstrom, S. P., Arends, J.J., Jacquin, M.F., and Woolsey, T.A. (1996). Postnatal development of mouse "whisker" thalamus: ventroposterior medial nucleus (VPM), barreloids, and their thalamocortical relay neurons. Somatosens. Mot. Res. 13, 307-322.

Zhang, D., Snyder, A. Z., Fox, M. D., Sansbury, M. W., Shimony, J. S., and Raichle, M. E. (2008). Intrinsic functional relations between human cerebral cortex and thalamus. $J$. Neurophysiol. 100, 1740-1748.

Zhang, D., Snyder, A. Z., Shimony, J. S., Fox, M. D., and Raichle, M. E. (2009). Noninvasive functional and structural connectivity mapping of the human thalamocortical system. Cereb. Cortex 20, 1187-1194.

Conflict of Interest Statement: The authors declare that the research was con- ducted in the absence of any commercial or financial relationships that could be construed as a potential conflict of interest.

Received: 05 February 2010; paper pending published: 01 March 2010; accepted: 06 April 2010; published online: 18 May 2010.

Citation: Fair DA, Bathula D, Mills KL, Dias TGC, Blythe MS, Zhang D, Snyder $A Z$, Raichle ME, Stevens AA, Nigg JT and Nagel BJ (2010) Maturing thalamocor- tical functional connectivity across development. Front. Syst. Neurosci. 4:10. doi: 10.3389/fnsys.2010.00010

Copyright (๑) 2010 Fair, Bathula, Mills, Dias, Blythe, Zhang, Snyder, Raichle, Stevens, Nigg and Nagel. This is an openaccess article subject to an exclusive license agreement between the authors and the Frontiers Research Foundation, which permits unrestricted use, distribution, and reproduction in any medium, provided the original authors and source are credited. 